\title{
Perfusão regional de membros no tratamento quimioterápico de melanoma: uma revisão de literatura
}

\author{
Lídia Trommer Marcos*, Djuli Milene Hermes \\ Centro Universitário Ritter dos Reis - UniRitter, Porto Alegre, RS, Brasil
}

Histórico do Artigo

Recebido em:

25/08/2019

Aceito em:

04/11/2019

\section{Palavras-chave:}

Perfusão Regional;

Melanoma;

Quimioterapia

Keywords:

Regional Perfusion;

Melanoma;

Chemotherapy

\begin{abstract}
RESUMO
O câncer de pele melanoma corresponde a cerca de $3 \%$ das neoplasias malignas na pele e é o tipo mais grave de câncer de pele. De todos os casos de melanoma cutâneo primário, $50 \%$ das lesões ocorrem em extremidades. O tratamento regional e concentrado tem a capacidade de diminuir as recidivas e melhorar o prognóstico do paciente e é feito em uma dose que chega a ser até 10 vezes a dose aplicada em tratamentos sistêmicos. Após mais de 60 anos de experiência com perfusão isolada de membros (PIM), foram publicados diversos estudos que indicam resultados geralmente favoráveis a utilização desta técnica. O presente trabalho tem por objetivo, analisar os resultados obtidos nos últimos 10 anos de publicações com relação a efetividade deste tratamento contra os tumores do tipo melanoma não passíveis de remoção cirúrgica e a repercussão com relação a toxicidade local no paciente portador de melanoma em membro isolado. Vinte artigos sobre PIM para o tratamento de melanoma foram analisados para este trabalho, totalizando 1800 procedimentos. Os dados relativos à resposta ao tratamento foram analisados em 19 artigos, totalizando 1563 procedimentos, com uma média de resposta ao tratamento de 85,6\%. A toxicidade local do membro tratado foi analisada em 16 artigos, totalizando 1501 procedimentos, sendo que em média $96 \%$ dos procedimentos realizados apresentaram um grau de toxicidade igual ou menor a III na escala de Wieberdink.
\end{abstract}

Isolated limb perfusion in melanoma chemotherapy treatment: a literature review

\section{ABSTRACT}

Melanoma skin cancer is accounting for about $3 \%$ of malignant neoplasms of the skin and is the most serious type of skin cancer. Of all cases of primary cutaneous melanoma, $50 \%$ of lesions occur in the extremities. Regional and concentrated treatment has the ability to decrease relapses and improve patient prognosis and is done at a dose that is up to 10 times the dose applied in systemic treatments. After more than 60 years of experience with isolated limb perfusion (ILP), several studies have been published indicating results generally favorable to the use of this technique. The present work aims to analyze the results obtained in the last 10 years of publications about the effectiveness of this treatment against non-surgically removable melanoma tumors and the repercussions regarding local toxicity in the patient with isolated limb melanoma. Twenty articles on ILP for melanoma treatment were analyzed for this work, totaling 1800 procedures. Data on treatment response were analyzed in 19 articles, totaling 1563 procedures, with an average treatment response of $85.6 \%$. The local toxicity of the treated limb was analyzed in 16 articles, totaling 1501 procedures, and on average $96 \%$ of the procedures performed had a degree of toxicity equal to or less than III on the Wieberdink scale.

\section{Introdução}

O câncer de pele melanoma corresponde a cerca de $3 \%$ das neoplasias malignas na pele e é o tipo mais grave de câncer de pele. Em 2018 o INCA (Instituto Nacional do Câncer) estimou que haveriam 6.260 novos casos de melanoma no Brasil, sendo 2.920 em homens e 3.340 em mulheres (1). De todos os casos de melanoma cutâneo primário, $50 \%$ das lesões ocorrem em extremidades (2), sendo que $30 \%$ ocorrem nos membros inferiores e $20 \%$ nos membros superiores (3). Após a retirada do tumor primário podem ocorrer recidivas no mesmo local, em regiões próximas ou em áreas mais distantes do

\footnotetext{
* Autor correspondente: lidiatrommer@gmail.com (Marcos L.T.)
} 
corpo do paciente, porém as metástases em trânsito (lesões múltiplas na derme ou tecido subcutâneo superficial entre a lesão primária e a cadeia linfonodal regional) são as que merecem maior destaque, já que ocorrem em até $11 \%$ dos pacientes com melanoma (4).

A circulação extracorpórea possui muitas utilidades além da cirurgia cardíaca, e um de seus usos mais recentes é a perfusão regional no tratamento do câncer. Tendo sido desenvolvida por Creech e Krementz, em 1956, a perfusão de um membro ou órgão isolado permite que estes sejam infundidos com drogas quimioterápicas e hipertermia sem expor o restante do organismo a esses medicamentos e seus efeitos colaterais. Ou seja, o objetivo da perfusão isolada de membros (PIM) é obter o máximo de resposta de morte tumoral com o mínimo de toxicidade sistêmica (5). Essa técnica utiliza o mesmo equipamento de uma circulação extracorpórea convencional (CEC) e, dessa forma, realiza a oxigenação e o bombeamento de sangue arterial para o membro ou órgão que está sendo tratado, porém de forma a excluí-lo, temporariamente, da circulação do resto do corpo (6).

Outras opções de tratamento podem ser altamente mutiladoras dependendo do tumor, enquanto que a perfusão regional fornece um resultado mais funcional se utilizado de forma conjunta com a quimioterapia convencional e a radioterapia (6). Quando comparadas PIM e amputação, a primeira apresenta uma taxa de sobrevivência, nos 5 anos seguintes, de $17 \%$ até $52 \%$, enquanto que a segunda de $7 \%$ até $28 \%$ (7).

A técnica da perfusão regional consiste na canulação de uma artéria e de uma veia, na raiz do membro portador do tumor, e no isolamento do membro através da dissecção de ramos secundários de artérias, e aplicação de um torniquete acima do nível das cânulas (6). Geralmente são utilizadas as veias femorais para as pernas e as veias axilares para os braços (4). Após o isolamento do membro é de vital importância que se verifique se não existe nenhum vazamento para a circulação sistêmica, ou seja, se o membro está realmente isolado (8).

Os principais tipos de tumores em que a perfusão regional é indicada são os melanomas, os sarcomas ósseos e os sarcomas de partes moles. No caso dos melanomas, a PIM é indicada pelos oncologistas, quando se trata de uma lesão em um membro específico, em estágio mais avançado, em que não existe possibilidade de remoção cirúrgica ou quando o paciente não aceita a amputação do membro afetado.

A PIM é indicada em pacientes que apresentam disseminação maciça de câncer do tipo melanoma em membros superiores ou inferiores, sem disseminação sistêmica. A American Cancer Society aponta que a PIM é a escolha no caso de pacientes com melanoma em estágio III quando os tumores estiverem restritos aos membros inferiores ou superiores. Os melanomas em estágio III são aqueles em que o tumor pode ter qualquer espessura (com ou sem ulceração),porém o câncer já se espalhou para áreas próximas como gânglios linfáticos e linfonodos ou outros lugares da pele a não mais de 2 centímetros de distância do tumor primário, sem que tenha se espalhado para outros órgãos (9).

O tratamento regional e concentrado tem a capacidade de diminuir as recidivas e melhorar o prognóstico do paciente e é feito, geralmente, com Melfalan associado ou não com TNFa (fator de necrose tumoral alfa), em uma dose que chega a ser até 10 vezes a dose aplicada em tratamentos sistêmicos (10)(7). Após a PIM, é realizada uma lavagem com solução cristaloide. Dessa forma, quando o garrote é retirado e todo o quimioterápico já foi removido pela lavagem, a toxicidade sistêmica pode ser considerada negligenciável (4).

Após mais de 60 anos de experiência com PIM, foram publicados diversos estudos que indicam resultados geralmente favoráveis a utilização desta técnica. Sendo assim, o presente trabalho tem por objetivo, através de uma revisão de literatura sistemática, analisar os resultados obtidos nos últimos 10 anos, com relação à efetividade deste 
tratamento contra os tumores do tipo melanoma não passíveis de remoção cirúrgica e a repercussão com relação à toxicidade local no paciente portador de melanoma em membro isolado.

\section{Materiais e métodos}

Para realizar a revisão de literatura, foram pesquisadas as bases de dados Pubmed, Scielo e Lilacs com as palavras chaves anteriormente verificadas e descritas no Descritores em Ciências da Saúde (DeCS). As palavras chave de busca foram: Perfusão Regional, Melanoma, Quimioterapia.

Foram incluídos artigos apenas em língua inglesa que estavam entre o período de janeiro de 2010 até dezembro de 2019, totalizando dez anos, em que constavam como assuntos principais o tratamento do câncer do tipo melanoma (sendo a PIM um dos tipos de tratamentos) ou aqueles em que perfusão isolada de membros fosse o foco. Em ambos os casos constavam dados relativos à classificação quanto à remissão e/ou relativos à classificação quanto à toxicidade.

Foram excluídos artigos que estavam escritos em outras línguas que não aquela préestipulada, artigos publicados que não estavam dentro do período estabelecido, artigos que não envolvessem seres humanos, artigos que tratassem de perfusão de quimioterápicosque não de forma isolada em membros superiores e inferiores, artigos que tratassem de outros tipos de tumores. Também foram excluídos artigos que tratavam de infusão regional isolada de membros, por ser uma técnica diferente da perfusão regional isolada de membros.

Para serem incluídos nesta revisão os artigos deveriam utilizar o critério RECIST (Response Evaluation Criteria in Solid Tumors) quanto a eficácia do tratamento foi utilizado.O RECIST considera os seguintes parâmetros: resposta completa (RC) o desaparecimento total das lesões, resposta parcial (RP) redução de pelo menos $50 \%$ da soma dos diâmetros das lesões-alvo, doença progressiva o aumento de pelo menos $25 \%$ da soma dos diâmetros das lesões-alvo e doença estável não ocorre mudança suficiente pra se encaixar em outra classificação (11).

Em relação a toxicidade regional os artigos deveriam utilizar o sistema proposto por Wieberdink que classifica em 5 graus a possível reação. Os graus do sistema de Wieberdink são: I - sem reação, II - Pequeno eritema ou edema, III - eritema ou edema considerável com algumas bolhas, IV - epidermolise excessiva ou danos aos tecidos mais profundos causando distúrbio funcional ou síndrome compartimental, V - reação severa que pode necessitar de amputação do membro (12).

Após a pesquisa inicial nas bases de dados com as palavras chave, o período, a língua e os sistemas escolhidos foram encontrados 150 artigos. Destes, 130 artigos foram excluídos por não se enquadrarem nos critérios previamente estabelecidos e o restante (20 artigos) foram analisados conforme o conteúdo apresentado, sendo 4 artigos sobre a resposta ao tratamento (dois deles avaliaram a toxicidade, porém dois utilizaram metodologia diferente da abordada nesta revisão de literatura e outro não fez a separação dos dados relativos à melanoma e sarcoma de partes moles, sendo considerados apenas os dados relativos à resposta ao tratamento), 1 artigo sobre a toxicidade e 15 artigos sobre ambos, resposta ao tratamento e toxicidade. 
Figura 1 - Metodologia para exclusão/inclusão dos artigos na revisão.

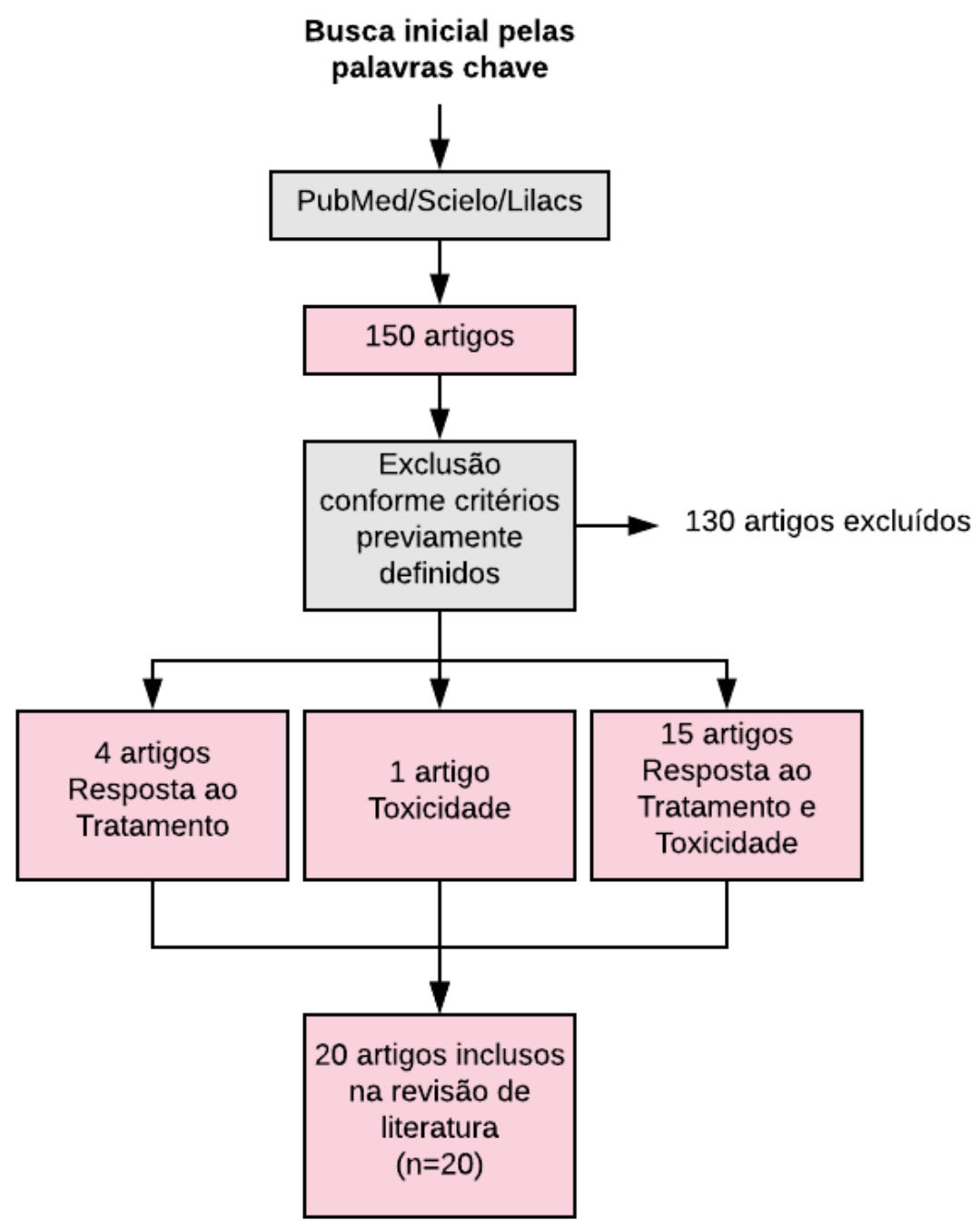

Posteriormente, foi efetuada leitura integral dos artigos que estavam dentro das especificações detalhadas acima observando seus resultados e discussões para análise dos mesmos e realizada a tabulação dos dados de forma a facilitarem aanálise quanto à remissão dos tumores e/ou toxicidade do tratamento, conforme a Tabela 1. A análise deste trabalho foi realizada através de compilação das informações em Word® e tratamento em Excel® na versão do Office® 2016.

\section{Resultados e discussão}

A PIM pode ser considerada a técnica de escolha no controle locorregional em pacientes que apresentam disseminação maciça em membros superiores ou inferiores, sem disseminação sistêmica de câncer do tipo melanoma em estágio III. Contudo, não existe, ainda, padronização quanto à utilização da técnica. Dentre os fatores que contribuem para esta falta de padronização é possível destacar: os diferentes regimes quimioterápicos utilizados nos estudos realizados (tanto quanto ao tipo de quimioterápicos utilizados, como quanto a repetição ou não do tratamento no mesmo local), a heterogeneidade dos grupos de pacientes atendidos e, principalmente, o elevado custo e conhecimento desta técnica, que dificulta sua utilização.

Vinte artigos sobre perfusão isolada de membros para o tratamento de melanomas localizados em membros isolados (superiores e inferiores) foram analisados para a 
realização deste trabalho, totalizando 1800 procedimentos ao longo dos últimos 10 anos de publicações, resultando em uma média de 2 artigos publicados por ano.

A tabela a seguir sumariza os vinte artigos inclusos nesta revisão de literatura, bem como o número de procedimentos/pacientes avaliados em cada um deles, o que foi analisado em cada um dos artigos citados, onde " $\mathrm{R}$ " representa resposta ao tratamento $\mathrm{e}$ " $T$ " representa toxicidade regional.

Tabela 1- Estudos sobre perfusão isolada de membros entre 2010 e 2019.

\begin{tabular}{|c|c|c|c|c|}
\hline & Estudos & Pacientes & PIMs avaliados & $\begin{array}{l}\text { O que foi } \\
\text { avaliado }\end{array}$ \\
\hline 1 & Alexander et al., 2010 & 90 pacientes & 90 PIMs & $\mathrm{Re} \mathrm{T}$ \\
\hline 2 & Bagge et al., 2016 & 50 pacientes & $\begin{array}{c}50 \text { PIMs (resposta ao } \\
\text { tratamento avaliada em } \\
49 \text { PIMs) }\end{array}$ & $\mathrm{Re} \mathrm{T}$ \\
\hline 3 & Boesch et al., 2010 & 145 pacientes & 145 PIMs & $\mathrm{R} \mathrm{e} \mathrm{T}$ \\
\hline 4 & Deroose et al., 2011 & 105 pacientes & 118 PIMS realizados & $\mathrm{Re} \mathrm{T}$ \\
\hline 5 & Deroose et al., 2012 & 148 pacientes & 167 PIMs & $\mathrm{R}$ e $\mathrm{T}$ \\
\hline 6 & Deroose et al., 2015 & 32 pacientes & 37 PIMs & $\mathrm{Re} \mathrm{T}$ \\
\hline 7 & Dossett et al., 2016 & 109 pacientes & 109 PIMs & $\mathrm{Re} \mathrm{T}$ \\
\hline 8 & $\begin{array}{l}\text { Hoekstra; Veerman; } \\
\text { Van Ginkel, } 2014\end{array}$ & $\begin{array}{l}60 \text { pacientes (resposta } \\
\text { ao tratamento avaliada } \\
\text { em } 57 \text { pacientes) }\end{array}$ & 60 PIMs & $\mathrm{Re} \mathrm{T}$ \\
\hline 9 & Lidsky et al., 2013 & 73 pacientes & 73 PIMs & $\mathrm{R}$ \\
\hline 10 & Madu et al., 2017 & $\begin{array}{l}91 \text { pacientes ( } 88 \\
\text { pacientes avaliados } \\
\text { para resposta ao } \\
\text { tratamento) }\end{array}$ & 91 PIMs & $\mathrm{Re} \mathrm{T}$ \\
\hline 11 & $\begin{array}{l}\text { Nyström Wendt; } \\
\text { Mattsson; Olofsson } \\
\text { Bagge, } 2017\end{array}$ & 193 pacientes & 193 PIMs & $\mathrm{T}$ \\
\hline 12 & Pace et al., 2011 & $\begin{array}{l}91 \text { pacientes (56 } \\
\text { pacientes avaliados } \\
\text { para resposta ao } \\
\text { tratamento) }\end{array}$ & 91 PIMs & $\mathrm{Re} \mathrm{T}$ \\
\hline 13 & Papadia et al., 2013 & $\begin{array}{l}17 \text { pacientes ( } 16 \\
\text { pacientes avaliados } \\
\text { para a resposta ao } \\
\text { tratamento) }\end{array}$ & 17 PIMs & $\mathrm{R} \mathrm{e} \mathrm{T}$ \\
\hline 14 & Paulsen et al., 2014 & 84 pacientes & 84 PIMs & $\mathrm{R} \mathrm{e} \mathrm{T}$ \\
\hline 15 & Raymond et al., 2011 & 60 pacientes & 60 PIMs & $\mathrm{R}$ \\
\hline 16 & Rossi et al., 2010 & 112 pacientes & 112 PIMs & $\mathrm{Re} \mathrm{T}$ \\
\hline 17 & Sharma et al., 2012 & 73 pacientes & 73 PIMs & $\mathrm{R}$ \\
\hline 18 & Smith et al., 2015 & 93 pacientes & 93 PIMs & $\mathrm{R}$ \\
\hline 19 & Smith et al., 2018 & $\begin{array}{c}109 \text { pacientes (108 } \\
\text { pacientesavaliados } \\
\text { quanto à resposta ao } \\
\text { tratamento) }\end{array}$ & 109 PIMs & $\mathrm{Re} \mathrm{T}$ \\
\hline 20 & Stamatiou et al., 2017 & 28 pacientes & 28 PIMs & $\mathrm{Re} \mathrm{T}$ \\
\hline
\end{tabular}

Os artigos analisados para esta revisão de literatura utilizaram diferentes regimes quimioterápicosna perfusão dos membros afetados, tendo os regimes quimioterápicos variado tanto entre os estudos como dentro de um mesmo estudo. Foram utilizados seis regimes quimioterápicos distintos, porém todos tiveram o Melfalan como base do tratamento. 
Estudo realizado em 2006 não encontrou vantagens quanto a utilização combinada de Melfalan + TNF em relação à utilização de Melfalan sozinho (32). Porém, outro estudo do mesmo ano aponta que as respostas completas ao tratamento aumentam de uma faixa entre $40 \%$ e $82 \%$ para uma faixa entre $59 \%$ e $85 \%$ quanto é utilizada a combinação Melfalan + TNF (33). Desta forma, assim como nos artigos analisados nesta revisão, ainda não se tem uma definição quanto a utilização dos diferentes regimes quimioterápicos. A tabela seguinte traz os estudos analisados juntamente com a quimioterapia utilizada em cada um deles.

Tabela 2- Regime quimioterápico nos estudos sobre perfusão isolada de membros entre 2010 e 2019.

\begin{tabular}{|c|c|c|}
\hline \multicolumn{2}{|r|}{ Estudo } & Regime Quimioterápico \\
\hline 1 & Alexander et al., 2010 & $\begin{array}{c}\text { Melfalan, Melfalan + TNF, Melfalan + TNF } \\
+ \text { Interferon }\end{array}$ \\
\hline 2 & Bagge et al., 2016 & Melfalan, Melfalan + TNF \\
\hline 3 & Boesch et al., 2010 & Melfalan + Dactinomycin \\
\hline 4 & Deroose et al., 2011 & Melfalan + TNF \\
\hline 5 & Deroose et al., 2012 & Melfalan + TNF \\
\hline 6 & Deroose et al., 2015 & Melfalan + TNF \\
\hline 7 & Dossett et al., 2016 & Melfalan + Actinomycin-D \\
\hline 8 & Hoekstra; Veerman; Van Ginkel, 2014 & Melfalan, Melfalan + TNF \\
\hline 9 & Lidsky et al., 2013 & Melfalan, Melfalan + Dactinomycin \\
\hline 10 & Madu et al., 2017 & Melfalan, Melfalan + TNF \\
\hline 11 & Nyström Wendt; Mattsson; Olofsson Bagge, 2017 & Melfalan \\
\hline 12 & Pace et al., 2011 & Melfalan \\
\hline 13 & Papadia et al., 2013 & L19 + Melfalan + TNF \\
\hline 14 & Paulsen et al., 2014 & Melfalan, Melfalan + TNF \\
\hline 15 & Raymond et al., 2011 & Melfalan \\
\hline 16 & Rossi et al., 2010 & Melfalan, Melfalan + TNF \\
\hline 17 & Sharma et al., 2012 & Melfalan + Dactinomycin \\
\hline 18 & Smith et al., 2015 & Melfalan + TNF \\
\hline 19 & Smith et al., 2018 & Melfalan + TNF \\
\hline 20 & Stamatiou et al., 2017 & Melfalan + TNF \\
\hline
\end{tabular}

Os dados relativos à resposta ao tratamento foram analisados em 19 artigos, totalizando 1563 procedimentos de PIM, com uma média de resposta ao tratamento de $85,6 \%$ (em uma faixa entre $77,7 \%$ e $94,6 \%$ ), sendo $56,1 \%$ (em uma faixa entre $25 \%$ e $89,2 \%$ ) de respostas completas e $29,5 \%$ (em uma faixa entre $5,4 \%$ e $62,5 \%$ ) de respostas parciais. Os dados encontrados na análise dos artigos corroboram com o que é apontado na literatura internacional sobre o assunto, que aponta uma média de resposta ao tratamento de $90,35 \%$ (em uma faixa entre $64 \%$ e $100 \%$ ) e uma média de resposta completa de $46,5 \%$ (variando entre $25 \%$ e $76 \%$ ) (5). Os dados relativos à doença progressiva, à doença estávele à não resposta ao tratamento não foram analisados nesta revisão. A tabela a seguir traz os 19 artigos que traziam dados quanto a resposta ao tratamento e seus respectivos resultados. 
Tabela 3 - Resposta ao tratamento nos estudos sobre perfusão isolada de membros entre 2010 e 2019.

\begin{tabular}{|r|r|r|r|r|}
\hline \multicolumn{1}{|c|}{ Estudos } & RC + RP & \multicolumn{1}{|c|}{ RC } & \multicolumn{1}{|c|}{ RP } \\
\hline $\mathbf{1}$ & Alexander et al., 2010 & $95 \%$ & $69 \%$ & $26 \%$ \\
\hline $\mathbf{2}$ & Bagge et al., 2016 & $77,6 \%$ & $61,2 \%$ & $16,3 \%$ \\
\hline $\mathbf{4}$ & Desch et al., 2010 & $80,7 \%$ & $62,8 \%$ & $17,9 \%$ \\
\hline $\mathbf{5}$ & Deroose et al., 2011 & $93,2 \%$ & $67,8 \%$ & $25,4 \%$ \\
\hline $\mathbf{6}$ & Deroose et al., 2012 2015 & $89 \%$ & $61 \%$ & $28 \%$ \\
\hline $\mathbf{7}$ & Dossett et al., 2016 & $86 \%$ & $65 \%$ & $21 \%$ \\
\hline $\mathbf{8}$ & Hoekstra; Veerman; Van Ginkel, 2014 & $80 \%$ & $60 \%$ & $19 \%$ \\
\hline $\mathbf{9}$ & Lidsky et al., 2013 & $90 \%$ & $45 \%$ & $45 \%$ \\
\hline $\mathbf{1 0}$ & Madu et al., 2017 & $78,1 \%$ & $49,3 \%$ & $28,8 \%$ \\
\hline $\mathbf{1 2}$ & Pace et al., 2011 & $81 \%$ & $47 \%$ & $34 \%$ \\
\hline $\mathbf{1 3}$ & Papadia et al., 2013 & $94,6 \%$ & $89,2 \%$ & $5,4 \%$ \\
\hline $\mathbf{1 4}$ & Paulsen et al., 2014 & $87,5 \%$ & $25 \%$ & $62,5 \%$ \\
\hline $\mathbf{1 5}$ & Raymond et al., 2011 & $85 \%$ & $42 \%$ & $43 \%$ \\
\hline $\mathbf{1 6}$ & Rossi et al., 2010 & $83,4 \%$ & $56,6 \%$ & $26,7 \%$ \\
\hline $\mathbf{1 7}$ & Sharma et al., 2012 & $90,1 \%$ & $51,4 \%$ & $38,7 \%$ \\
\hline $\mathbf{1 8}$ & Smith et al., 2015 & $78,1 \%$ & $49,3 \%$ & $28,8 \%$ \\
\hline $\mathbf{1 9}$ & Smith et al., 2018 & $81,8 \%$ & $45,2 \%$ & $36,6 \%$ \\
\hline $\mathbf{2 0}$ & Stamatiou et al., 2017 & $82,4 \%$ & $43,5 \%$ & $38,9 \%$ \\
\hline
\end{tabular}

A tabela 3 mostra as respostas ao tratamento (respostas completas + respostas parciais), bem como a resposta média entre os artigos. Dessa forma, é possível verificar que em nenhum dos estudos analisados a resposta foi inferior a $77 \%$, tendo se mantido em uma faixa relativamente constante. O que merece destaque, no entanto, é a composição da resposta ao tratamento, pois houve grande variação entre a porcentagem representada pelas respostas parciais e a porcentagem representada pelas completas entre os estudos.

A toxicidade local do membro tratado foi analisada em 16 artigos, totalizando 1501 procedimentos de PIM, sendo que, em média, 96\% (em uma faixa entre $89 \%$ e 100\%) dos procedimentos realizados apresentaram um grau de toxicidade igual ou menor a III na escala de Wieberdink. Como os graus da escala de Wieberdink se dividem em cinco diferentes graus (I - sem reação, II - pequeno eritema ou edema, III - eritema ou edema considerável com algumas bolhas, IV - epidermolise excessiva ou danos aos tecidos mais profundos causando distúrbio funcional ou síndrome compartimental, V - reação severa que pode necessitar de amputação do membro) é possível verificar que na maioria dos casos a toxicidade local não atingiu níveis em que o dano tecidual fosse severo e irreversível (graus IV e V).

Alguns artigos agruparam seus resultados nos graus de toxicidade de Wieberdink, juntando os graus I-II ou II-III ou IV-V o que impossibilitou uma análise da toxicidade local grau a grau, com isso a alternativa encontrada foi agrupar os graus leve e moderado (de I a III na escala de Wieberdink) e os graus grave e severo (de IV a V na escala de Wieberdink) para que a análise pudesse ser realizada.

O gráfico a seguir mostra a relação entre as reações leves e moderadas (de I a III na escala de Wieberdink) e as reações graves e severas (de IV a V na escala de Wieberdink). É possível visualizar claramente o número maciço de reações mais brandas (variando entre $89 \%$ e $100 \%$ ) em relação ao pequeno percentual de reações mais graves (variando entre $0 \%$ e $11 \%$ ). 
Figura 2 - Toxicidade local no membro tratado.

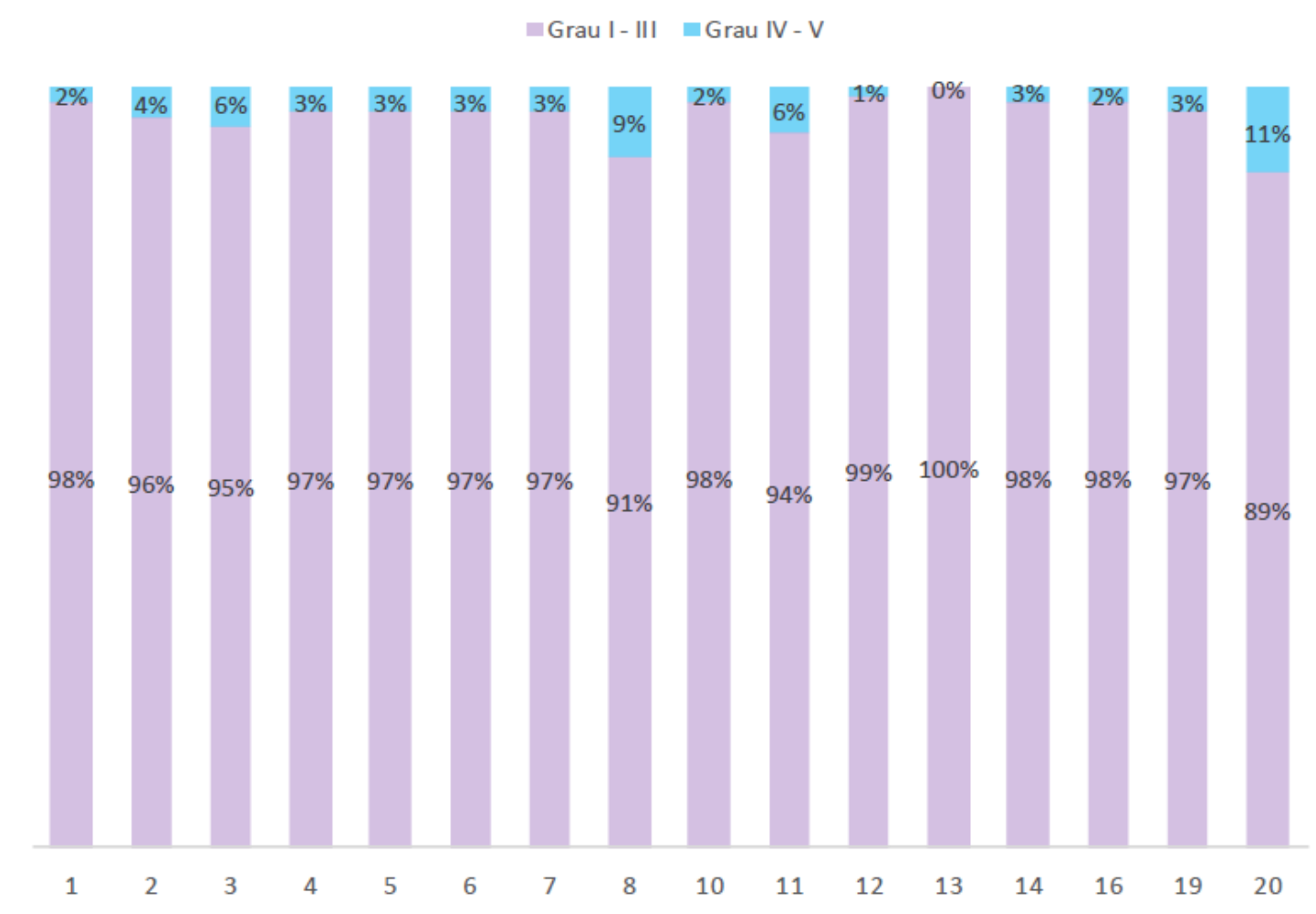

A baixa toxicidade local encontrada por esta análise é corroborada por estudo realizado em 2010 que aponta que a média de toxicidade local em grau II foi de 73,53\%, em grau III de $17,10 \%$, em grau IV 2,00\% e em grau V 0,65\% (5).

\section{Considerações finais}

Os artigos analisados e inclusos nesta revisão de literatura, em sua maioria,abordaram e discutiram os resultados com relação à resposta ao tratamento fazendo correlações entre tipo de resposta ao tratamento (total, completa, parcial, sem resposta, doença progressiva, doença estável) e o aumento da sobrevida média ou o tempo livre de metástases do mesmo gênero. Tendo em vista que esse tipo de tratamento tem por objetivo a melhora na qualidade de vida do paciente, reduz a alternativa da provável amputação do membro ou a convivência com sérias reações teciduais e o espalhamento mais rápido da doença. A outra correlação feita e que merece destaque é a toxicidade local resultante do tratamento versus efetividade deste tratamento, no sentido de justificar que o procedimento é relativamente seguro tendo em vista a alta porcentagem de resposta ao tratamento e o baixo índice de graus mais altos de toxicidade.

A análise realizada no presente trabalho evidencia os resultados encontrados pelos 19 artigos inclusos nesta revisão, uma vez que em todos os artigos analisados foi apresentado um alto índice de resposta ao tratamento, em torno de $86 \%$, com um baixo índice de toxicidade local em níveis mais altos, em torno de $4 \%$. O problema em relação a toxicidade é que apesar de acontecer, geralmente, em graus mais baixos, ela está presente praticamente na totalidade dos pacientes tratados com PIM.

Não foi verificada nenhuma correlação entre o grau de toxicidade local e a resposta ao tratamento nos artigos analisados.

Apesar da resposta ao tratamento ter sido bastante elevada e consistente nos artigos analisados, seriam necessários novos estudos de forma a verificar o motivo da faixa tão ampla de variação entre respostas completas e parciais. 
Uma limitação na análise realizada foram os diferentes tipos de regimes quimioterápicos utilizados nos estudos, tendo sido observada a utilização de Melfalan, Melfalan + TNF, Melfalan + TNF + Interferon, Melfalan + Dactinomicina, Melfalan + Actinomicina-D, Melfalan + TNF + L19. Com isso, não foi possível verificar como foi a resposta aos diferentes regimes e a toxicidade de cada um deles. Outra limitação da pesquisa foi o agrupamento dos resultados da toxicidade local de Wieberdink em alguns dos artigos impossibilitando que fosse realizada uma análise grau a grau, o que daria uma ideia mais precisa desse efeito colateral.

O melanoma é um tipo de câncer que vem crescendo nas últimas décadas em todo o mundo e que possui altas taxas de recuperação total, mesmo em estados mais avançados onde a cirurgia e excisão da lesão não são possíveis. Com a técnica de PIM a qualidade de vida dos pacientes aumenta de forma considerável, porém ainda é uma técnica de alto custo e que necessita de profissionais bastante especializados.

\section{Referências}

1. INCA. Câncer de pele melanoma [Internet]. Câncer de pele melanoma. 2018 [citado 6 de abril de 2019]. Available at: https://www.inca.gov.br/tipos-de-cancer/cancer-de-pele-melanoma

2. Han D, Beasley GM, Tyler DS, Zager JS. Minimally invasive intra-arterial regional therapy for metastatic melanoma: isolated limb infusion and percutaneous hepatic perfusion. Expert Opin Drug Metab Toxicol 2011;7(11):1383-94.

3. Hoekstra HJ, Veerman K, Van Ginkel RJ. Isolated limb perfusion for in-transit melanoma metastases: Melphalan or TNF-melphalan perfusion? J Surg Oncol 2014;109(4):338-47.

4. Gabriel E, Skitzki J. The role of regional therapies for in-transit melanoma in the era of improved systemic options. Cancers (Basel) 2015;7(3):1154-77.

5. Moreno-Ramirez D, de la Cruz-Merino L, Ferrandiz L, Villegas-Portero R, Nieto-Garcia A. Isolated Limb Perfusion for Malignant Melanoma: Systematic Review on Effectiveness and Safety. Oncologist 2010;15(4):416-27.

6. Souza ML, Elias DO. Fundamentos da Circulação Extracorpórea [Internet]. $2^{\circ}$ ed. Rio de Janeiro: Centro Editorial Alfa Rio; 2006. 828 p.

7. Nan Tie E, Henderson MA, Gyorki DE. Management of in-transit melanoma metastases: a review. ANZ J Surg 2019;89(6):647-652.

8. Testori A, Verhoef C, Kroon HM, Pennacchioli E, Faries MB, Eggermont AMM, et al. Treatment of melanoma metastases in a limb by isolated limb perfusion and isolated limb infusion. J Surg Oncol 2011;104(4):397-404.

9. American Cancer Society. Treatment of Melanoma Skin Cancer, by Stage [Internet]. 2019 [citado 12 de outubro de 2019]. Available at: https://www.cancer.org/cancer/melanoma-skin-cancer/treating/bystage.html

10. Tejada VF dos S, Rodrigues O. Neoplasias malignas de pele: revisão bibliográfica com ênfase à abordagem cirúrgica. Vittalle 2009;21(1):73-85.

11. Miller AB, Hoogstraten B, Staquet M, Winkler A. Reporting Results of Cancer Treatment. Cancer. 1981;47(C):207-14.

12. Wieberdink J, Benckhuysen C, Braat RP, Van Slooten EA, Olthuis GAA. Dosimetry in isolation perfusion of the limbs by assessment of perfused tissue volume and grading of toxic tissue reactions. Eur J Cancer Clin Oncol 1982;18(10):905-10.

13. Alexander HR, Fraker DL, Bartlett DL, Libutti SK, Steinberg SM, Soriano P, et al. Analysis of factors influencing outcome in patients with in-transit malignant melanoma undergoing isolated limb perfusion using modern treatment parameters. J Clin Oncol 2010;28(1):114-8.

14. Bagge ASL, Ben-Shabat I, Belgrano V, Olofsson Bagge R. Health-Related Quality of Life for Patients Who have In-Transit Melanoma Metastases Treated with Isolated Limb Perfusion. Ann Surg Oncol 2016;23(6):2062-9.

15. Boesch CE, Meyer T, Waschke L, Merkel S, Goehl J, Hohenberger W, et al. Long-term outcome of 
hyperthermic isolated limb perfusion (HILP) in the treatment of locoregionally metastasised malignant melanoma of the extremities. Int J Hyperth 2010;26(1):16-20.

16. Deroose JP, Grünhagen DJ, Van Geel AN, De Wilt JHW, Eggermont AMM, Verhoef C. Long-term outcome of isolated limb perfusion with tumour necrosis factor-?? for patients with melanoma in-transit metastases. Br J Surg 2011;98(11):1573-80.

17. Deroose JP, Eggermont AMM, Van Geel AN, De Wilt JHW, Burger JWA, Verhoef C. 20 years experience of TNF-based isolated limb perfusion for in-transit melanoma metastases: TNF dose matters. Ann Surg Oncol 2012;19(2):627-35.

18. Deroose JP, Grünhagen DJ, Eggermont AMM, Verhoef C. Repeated isolated limb perfusion in melanoma patients with recurrent in-transit metastases. Melanoma Res 2015;25(5):427-31.

19. Dossett LA, Ben-Shabat I, Olofsson Bagge R, Zager JS. Clinical Response and Regional Toxicity Following Isolated Limb Infusion Compared with Isolated Limb Perfusion for In-Transit Melanoma. Ann Surg Oncol 2016;23(7):2330-5.

20. Lidsky ME, Turley RS, Beasley GM, Sharma K, Tyler DS. Predicting disease progression after regional therapy for in-transit melanoma. JAMA Surg 2013;148(6):493-8.

21. Madu MF, Deken MM, van der Hage JA, Jóźwiak K, Wouters MWJM, van Akkooi ACJ. Isolated Limb Perfusion for Melanoma is Safe and Effective in Elderly Patients. Ann Surg Oncol 2017;24(7):1997-2005.

22. Nyström Wendt A, Mattsson J, Olofsson Bagge R. Myoglobin does not predict local toxicity in isolated limb perfusion. Int J Hyperth 2017;33(6):679-83.

23. Pace M, Gattai R, Mascitelli EM, Millanta L. Results of isolated lower limb perfusion for locoregional advanced/recurrent melanoma using borderline true hyperthermia plus additional bolus of melphalan. A critical analysis of homogeneous cases. J Surg Oncol 2011;104(7):718-23.

24. Papadia F, Basso V, Patuzzo R, Maurichi A, Di Florio A, Zardi L, et al. Isolated limb perfusion with the tumor-targeting human monoclonal antibody-cytokine fusion protein L19-TNF plus melphalan and mild hyperthermia in patients with locally advanced extremity melanoma. J Surg Oncol 2013;107(2):1739 .

25. Paulsen IF, Chakera AH, Drejøe JB, Klyver H, Dahlstrøm K, Oturai PS, et al. Tumour response after hyperthermic isolated limb perfusion for locally advanced melanoma. Dan Med J 2014;61(1):1-5.

26. Raymond AK, Beasley GM, Broadwater G, Augustine CK, Padussis JC, Turley R, et al. Current trends in regional therapy for melanoma: Lessons learned from 225 regional chemotherapy treatments between 1995 and 2010 at a single institution. J Am Coll Surg 2011;213(2):306-16.

27. Rossi CR, Pasquali S, Mocellin S, Vecchiato A, Campana LG, Pilati P, et al. Long-term results of melphalan-based isolated limb perfusion with or without low-dose TNF for in-transit melanoma metastases. Ann Surg Oncol 2010;17(11):3000-7.

28. Sharma K, Beasley G, Turley R, Raymond AK, Broadwater G, Peterson B, et al. Patterns of recurrence following complete response to regional chemotherapy for in-transit melanoma. Ann Surg Oncol 2012;19(8):2563-71.

29. Smith HG, Cartwright J, Wilkinson MJ, Strauss DC, Thomas JM, Hayes AJ. Isolated Limb Perfusion with Melphalan and Tumour Necrosis Factor $\alpha$ for In-Transit Melanoma and Soft Tissue Sarcoma. Ann Surg Oncol 2015;22:356-61.

30. Smith HG, Wilkinson MJ, Smith MJF, Strauss DC, Hayes AJ. The effect of age on outcomes after isolated limb perfusion for advanced extremity malignancies. Eur J Cancer 2018;100:46-54.

31. Stamatiou D, Ioannou C V., Kontopodis N, Michelakis D, Perisinakis K, Lasithiotakis K, et al. Hyperthermic isolated limb perfusion. The switch from Steinmann pins to Omni-tract assisted isolation. J Surg Res 2017;213:147-57.

32. Cornett WR, McCall LM, Petersen RP, Ross MI, Briele HA, Noyes RD, et al. Randomized multicenter trial of hyperthermic isolated limb perfusion with melphalan alone compared with melphalan plus tumor necrosis factor: American College of Surgeons Oncology Group trial Z0020. J Clin Oncol 2006;24(25):4196-201.

33. Grünhagen DJ, de Wilt JHW, van Geel AN, Eggermont AMM. Isolated limb perfusion for melanoma patients-a review of its indications and the role of tumour necrosis factor- $\alpha$. Eur J Surg Oncol 2006;32(4):371-80. 
Vittalle - Revista de Ciências da Saúde v. 31, n. 2 (2019) 77-87

EDITORA E GRÁFICA DA FURG

CAMPUS CARREIROS

CEP 96203900

editora@furg.br 